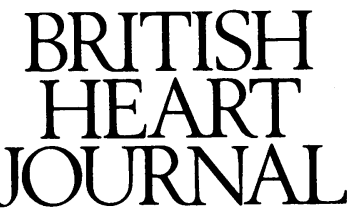

VIEWPOINT

\title{
Does angioplasty need on site surgical cover? A surgeon's view
}

\author{
D John Parker
}

Though angioplasty is an established technique for the management of coronary artery disease, there is still controversy about whether it needs on site surgical cover.

The aims of angioplasty and coronary artery bypass surgery in the management of coronary disease are similar, namely to relieve symptoms and in certain groups to improve prognosis, at the lowest risk and morbidity. Because the development of myocardial damage during both procedures significantly reduces long term survival, treatment strategies must not be based on short term results. Myocardial damage at the time of intervention and subsequently is important.

The principal risk of angioplasty is the development of acute myocardial ischaemia progressing to infarction owing to vessel occlusion with or without dissection. Increasing technical skills and improved equipment may reduce these complications but vessel occlusion is likely to remain the chief immediate complication of angioplasty.

The most reliable data on the true incidence of complications of angioplasty are more likely to come from registers than from individual hospitals. In the United States, the Second Generation 1985/6 Percutaneous Transluminal Coronary Angioplasty Register of the National Heart, Lung and Blood Institute (incorporating 1801 patients) showed a vessel occlusion rate of $4.9 \%$, an emergency coronary artery bypass rate of $3.5 \%$, a non-fatal myocardial infarction rate of $4.3 \%$, and an inhospital mortality of $1 \%$ (varying from $0.2 \%$ for single vessel disease to $2 \cdot 2 \%$ for triple vessel disease). ${ }^{1}$ The French registry for 1987 reviewed 2700 patients and recorded a $2 \cdot 1 \%$ infarction rate, a $1.7 \%$ urgent surgery rate, and $0.9 \%$ mortality. ${ }^{2}$

Not all patients in whom vessel occlusion develops need either further angioplasty or emergency surgery, particularly if there is no evidence of myocardial ischaemia or if the occluded vessel is very small. A further group of patients will have been advised to have angioplasty rather than surgery because disease in other organs puts them a higher sur- gical risk and in such patients a myocardial infarction may be the less dangerous option.

Clearly defined treatment strategies should be developed to prevent or limit myocardial damage once vessel occlusion occurs and there is evidence of myocardial ischaemia. Prompt action is required and the best solution is one achieved immediately by the angioplasty operator-but such attempts are successful in only about $50 \%$ of patients and are still associated with a considerable risk of myocardial infarction. More extended use of techniques such as autoperfusion dilatation catheters, stents, laser welding, or arthrectomy devices ${ }^{3}$ might reduce the need for emergency surgery, but they remain experimental. For patients who are haemodynamically unstable, the use of an intra-aortic balloon pump or percutaneous cardiopulmonary bypass may help to stabilise their condition but should not delay surgical intervention.

Given that surgery is important in the mangement of complications of angioplasty, how close should the operating theatre be to the catheterisation laboratory? It is important to plan for the worst outcome-which is cardiac arrest requiring continuing cardiopulmonary resuscitation. Once vessel occlusion and myocardial ischaemia have occurred and it is clear that further angioplasty techniques have failed, most patients should be transferred to the operating theatre as soon as possible. The effect of the length of the interval from occlusion to surgical revascularisation on subsequent infarction is not clear, largely because it is a non-homogeneous population; however, in large series rates of myocardial infarction in patients requiring emergency surgery varied from $11 \%$ to $46 \%{ }^{4}$ Furthermore, the extent of the infarction as well as its occurrence is important and the evidence of the effects of time to reperfusion from studies of thrombolysis indicates the need for rapid intervention.

In a series of 4142 patients in Eindhoven of whom 155 required emergency surgery, five were taken to theatre in cardiac arrest and 19 in cardiogenic shock and were revascularised 
in a median time of 103 minutes and the remaining patients in a median time of 135 minutes. ${ }^{5}$ Despite this rapid response time, $40^{\circ}{ }_{0}$ of both groups sustained a myocardial infarction. After a careful analysis the Eindhoven workers concluded that the complications of angioplasty remain unpredictable and they recommended continuing surgical cover.

Richardson et al reported on a selected series of 540 angioplasties from Belfast in which 12 patients required urgent surgery and were transferred $2.4 \mathrm{~km}$ to another hospital. The mean time to revascularisation was 268 minutes, similar to the time achieved in the receiving hospital. ${ }^{6}$ Such intervals are twice as long as in the Eindhoven study and most would view them as being unacceptably long. No information was given on the myocardial infarction rate in the surgical patients and in those who developed vessel occlusion but did not undergo surgery. The absence of these data seriously compromises the value of this study.

Prompt revascularisation can best be achieved in hospitals in which there are at least two operating theatres devoted entirely to cardiac surgery. Then emergencies can be handled quickly without seriously interrupting planned operations.

The earlier the patient reaches the theatre the greater the chance that the surgeon will use the internal mammary artery, which in the territory of the anterior descending coronary artery gives a better long term outcome.

Transfer of the patient to another hospital causes inevitable delay even with coordinated planning. A special report of a Joint American College of Cardiology and American Heart Association Task Force recommended that angioplasty should be undertaken at a hospital where an experienced cardiac surgical team was available for emergency surgery. ${ }^{7}$ This is also the view of the Society of Cardiothoracic Surgeons of Great Britain and Ireland and the International Society and Federation of Cardiology. ${ }^{8}$ In the United Kingdom there is no agreed policy on this issue and several units are undertaking angioplasty without on site surgical cover. All these units have or could develop close links with a major centre with surgical facilities.
Surely the best option is for the major centres to provide an angioplasty facility for cardiologists who do not have on site surgical cover but who do have the appropriate expertise and experience to undertake angioplasty? Such a policy should improve the results of angioplasty because it would concentrate practitioners of angioplasty into a limited number of hospitals and so increase their opportunities to interchange experience, increase contact with surgeons, and lead to a more comprehensive examination of the place of angioplasty and surgery in the management of coronary artery disease including the role of surgery in myocardial ischaemia induced by angioplasty.

It is more appropriate for cardiologists to travel by car than for patients with acute myocardial ischaemia (who perhaps will require cardiopulmonary resuscitation) to travel by ambulance. Angioplasty supported by on site surgical facilities is the correct policy for the early 1990 s and perhaps cardiologists should ask themselves where they as patients would prefer to have an angioplasty.

1 Holmes DR, Holubkov R, Vlietstra RE, et al. Comparison of complications during percutaneous transluminal coronary angioplasty from 1977-1981 and from 1985-1986: The National Heart, Lung and Blood Institute Percutaneous Transluminal Coronary Angioplasty Registry. J Am Coll Cardiol 1988;12:1149-55.

2 Gaspard PL, Didier B, Marco J, et al. What can we expect after coronary angioplasty? Immediate results: The French registry. Eur Heart $J$ 1989;10 (abstract suppl): 368.

3 Topol EJ. Emerging strategies for failed percutaneous transluminal coronary angioplasty. Am J Cardiol 1989;63: 249-50.

4 Talley JD, Jones EL, Weinstraub WS, King SB III Coronary artery bypass surgery after failed elective percoronary artery bypass surgery after failed elective percutaneous transluminal coronary angioplasty-
report. Circulation 1989;79(suppl I):I.126-I.31.

5 Bredee JJ, Bavinck JH, Berreklouw E, et al. Acute myocardial ischaemia and cardiogenic shock after percutaneous transluminal coronary angioplasty; risk factors for and results of emergency coronary bypass. Eur Heart J 1989 10(suppl H): 104-11.

6 Richardson SG, Morton P, Murtagh JG, O'Keeffe DB Murphy P, Scott ME. Management of acute occlusion during percutaneous transluminal coronary angioplasty: experience of complications in a hospital without on site facilities for cardiac surgery. $\mathrm{Br} M$ Med J 1990;300:355-8.

7 A Report of the American College of Cardiology/American Heart Association Task Force on Assessment of Diagnostic and Therapeutic Cardiovascular Procedures (Subcommittee on Percutaneous Transluminal Coronary Angioplasty. Guidelines for percutaneous transluminal coronary angioplasty. Circulation 1988;78:486-502.

8 Report of the Joint International Society and Federation of Cardiology/World Health Organisation Task Force in Coronary Angioplasty. Eur Heart J 1989;9:1034-45. 